\title{
Atribuições causais e nível educativo familiar na compreensão do desempenho escolar em alunos portugueses
}

\author{
Lúaia C. Miranda - InstitutoSuperior deEduraçãoeTrabalho, Poto Poturgal \\ L@indroS. Almida - UniversidadedoMinho Braca, Portugal \\ EvedyBonuhovith- UnivesidadeEstadual deCampinas, SãoPaulo, Brasil \\ AntónioR. Almida- Escda Básica eSeundánia deCdoricodeBasto, Braga, Poturgal \\ Sofia A. Abreu - Escda Básica eSecundánia deCdoricodeBasto Braca, Poturgal
}

\begin{abstract}
Resumo
Este artigo analisa o contributo específico do nível educativo familiar e das atribuições causais para o bom e fraco rendimento escolar dos alunos na explicação do seu desempenho nas disciplinas de Língua Portuguesa e de Matemática numa amostra de 2.082 alunos do ensino público de 11 escolas de Portugal, incluindo as Regiões Autónomas dos Açores e da Madeira. As atribuições causais foram avaliadas por meio do Questionário das Atribuições Causais para os Resultados Escolares (QARE). O nível educativo familiar foi estimado levando em conta o nível escolar do progenitor com maior habilitação escolar. As classificações obtidas pelos alunos nas disciplinas de língua portuguesa e matemática foram também examinadas. Os resultados apontam para correlações estatisticamente significativas, destacando as atribuições na capacidade para a explicação do rendimento escolar, situação que contrasta com a atribuição dos níveis de rendimento a variáveis externas ao aluno. A análise de regressão permite associar 34,5\% da variância no rendimento conjunto em Língua Portuguesa e em Matemática no ensino básico, assim como 21,3\% no ensino secundário, às dimensões atribucionais e ao nível educativo familiar. Implicações educacionais são derivadas a partir dos achados.
\end{abstract}

Palavasdhave Nível educativo familiar; A tribuições causais; Rendimento escolar; Língua portuguesa; Matemática.

\section{Causal attributions and educational level of the family in understanding school performance in Portuguese students}

\begin{abstract}
This paper analyzes the contribution of both family educational level and causal attributions in explaining students academic performance in Mathematics and Portuguese Language in a sample of 2.082 public school students from mainland Portugal and the Azores and Madeira regions. Causal attributions were assessed by the Causal Attributions Questionnaire for School Results (QARE). The family educational level was estimated taking into account the educational level of the parent with higher academic degree. The academic grades obtained by students in mathematics and portuguese language were also considered. The results show statistically significant correlations between variables. Internal causal attributions such as capacity and study methods were predominant to explain school performance in the sample and stood out in contrast to external attributions explanations. Regression analyses revealed that $34.5 \%$ of the variance in the combined achievement on Portuguese Language and Mathematics in primary education and $21.3 \%$ in secondary education could be explained by both the attributional dimensions and family education level. Some educational implications are present taking these data.
\end{abstract}

Keywards Family educational level; Causal attributions; Academic achievement.

\section{Atribuciones causales y nivel educativo familiar en la comprensión del desempeño escolar en alumnos portugueses}

\begin{abstract}
Resumen
Este artículo analiza la contribución específica del nivel educativo familiar y de las atribuciones causales para el buen y el flaco rendimiento escolar de los alumnos en la explicación de su desempeño en las disciplinas de Lengua Portuguesa y Matemáticas en una muestra de 2.082 alumnos de enseñanza pública de 11 escuelas de Portugal, incluyendo las Regiones Autónomas de Açores y Madeira. Las atribuciones causales fueron evaluadas por medio del "Questionário das Atribuições Causais para os Resultados Escolares" (QARE). El nivel educativo familiar fue estimado teniendo en cuenta el nivel escolar del progenitor con mayor habilitación escolar. Las clasificaciones obtenidas por los alumnos en las disciplinas de lengua portuguesa y matemáticas fueron también examinadas. Los resultados señalaron correlaciones estadísticamente significativas, destacando las atribuciones en la capacidad para la explicación del rendimiento escolar, situación que contrasta con la atribución de los niveles de rendimiento a variables externas al alumno. El análisis de regresión permite asociar 34.5\% de la variancia en el rendimiento conjunto de la Lengua Portuguesa y las Matemáticas en la enseñanza básica, así como 21.3\% en la enseñanza secundaria, a las dimensiones de atribución y al nivel educativo familiar. Implicaciones educacionales son derivadas a partir de los hallazgos.
\end{abstract}

Palabrasdave Nivel educativo familiar; Atribuciones causales; Rendimiento escolar; Lengua portuguesa; Matemáticas.

As inquietações com os fenômenos de exclusão social, principalmente a partir da segunda metade do século XX, problematizaram a instituição escolar e questionaram as suas práticas face às diferenças existentes na população discente. Os paradigmas que apontavam práticas pedagógicas que excluíam os alunos que se afastavam da norma foram postos em causa. Emergiram, então, as ideias da inclusão e de um tratamento diferenciado em função das necessidades educativas de cada aluno. Em consequência, 
protissionais e investigadores säo instados na compreensão dos fatores explicativos do sucesso dos alunos e na explicitação das medidas educativas que 0 possam promover, sobretudo nas sociedades em que a frequência escolar é obrigatória ou compulsiva (Almeida \& Roazzi, 1988; Degenszanjn, Roz \& Kotsubo, 2001; Mendonça, 2007).

A preocupação com o sucesso escolar, e a sua natureza multidimensional e multideterminada, justifica o estudo de variáveis associadas ao aluno, à prática pedagógica do professor, à rede de apoio familiar e às políticas educativas (Barbera, 2003; Formosinho, 1991; Guzzo, 2001; Mendonça, 2007; OECD, 2009). De acordo com a Organização para a Cooperação e D esenvolvimento Econômico (OECD , 2009), diversos são os indicadores para o sucesso escolar: (1) taxa de conclusão de cada ciclo e matrícula no ciclo seguinte; (2) perfil de competências dos alunos de 15 anos nas ciências, matemática e leitura; e (3) consequências sociais e laborais na idade adulta em alunos que não alcançaram uma preparação adequada.

Uma das variáveis importantes na análise do sucesso escolar prende-se à origem sociocultural do aluno. Os alunos provenientes de meio sociocultural desfavorecido provavelmente não disporão de uma base cultural necessária para triunfar na escola, acabando esta por ser acusada de algum efeito seletivo e reprodutivo das diferenças sociais nas certificações que outorga. $\mathrm{Na}$ verdade, os hábitos de vida, os modelos de interação familiar, as expetativas educativas em relação à aprendizagem dos filhos e a falta ou a baixa qualidade do apoio em relação às tarefas escolares, entre outros, influenciam adversamente 0 desenvolvimento cognitivo e psicossocial, assim como a aprendizagem escolar (Broman, Bien \& Schaugenessy, 1985; Eurydice, 1994; Jadue, 2002, 2003; Jadue, Galindo \& Navarro, 2005). O relatório da UNESCO (1996) refere que os pais com baixo nível socioeconômico, para além de utilizarem estratégias pouco eficazes nas interações com os seus filhos a propósito das atividades acadêmicas, também apresentam baixas expetativas face às mesmas. $\mathrm{O}$ baixo nível socioeconômico e educativo das famílias surge, assim, como um dos preditores mais consistentes dos problemas de desenvolvimento e de rendimento escolar (Pérez-Díaz, Rodriguez \& Sanchez, 2001).

Analisando as variáveis familiares, Formosinho (1991) sugere que o impacto é mais marcado pela escolaridade dos progenitores do que pelo seu nível socioeconômico. Os estudos de Mella e Ortiz (1999) e de Davis-Kean (2005) corroboram esta ideia, descrevendo as habilitações acadêmicas dos pais, e em particular das mães, como determinantes das expetativas escolares dos filhos. Esses estudos mostram que pais com maiores habilitaçöes acadëmicas investem mais na vida escolar dos seus filhos e acompanham-nos, por exemplo, quando estes apresentam dificuldades nas suas aprendizagens. Esses dados tendem a ser observados nos diferentes países e culturas, não sendo específicos dos países ocidentais (Dumka, Gonzales, Bonds \& Millsap, 2010; EnginD emir, 2009; Flouri \& Buchanan, 2004).

Procurando aprofundar o significado dos estudos disponíveis, as habilitações acadêmicas dos pais diferenciam os seus estilos e práticas educativas, e em particular o seu investimento no trabalho escolar dos filhos. Pais com mais formação acadêmica facilitam deliberadamente 0 desenvolvimento cognitivo e a autonomia das crianças, favorecem a sua autoestima, incentivam o esforço e os métodos de estudo, ajudam a fixar metas escolares, a apresentar atribuições causais apropriadas na explicação dos resultados acadêmicos e a desenvolver uma motivação intrínseca (D owning, Ollia \& Olivier, 1977; García \& Sánchez, 2005; G onzález-Pienda \& cols., 2002).

Uma das formas de analisar o impacto dos pais na aprendizagem escolar dos alunos prende-se às percepções, expetativas e aspirações que os pais expressam diante da escolarização e dos desempenhos escolares dos filhos (Ames, 1993; Deslandes, 2001; Garfaella, Gargallo \& Sánchez, 2001; Jacobs \& Eccles, 1992; Martin, Ranson \& Tall, 1997; McGrath \& Repetti, 2000; Reeve, Deci \& Ryan, 2004; Symeou, 2005). Tais vivências dos pais, como aliás dos seus professores, são inferidas e integradas no autoconceito de competência dos alunos e nas suas atribuições causais para os resultados escolares atingidos, refletindo-se na autoestima e na motivação acadêmica dos alunos (Gonzalez-Pienda \& cols., 2002). Nas últimas décadas, a investigação em psicologia educacional salienta que, a par das aptidões cognitivas, o sucesso escolar dos alunos encontra-se significativamente associado às suas características motivacionais (Almeida, 1996; Barros, 1996; Boruchovitch, 1994; Gonzalez-Pienda \& cols., 2002; Pintrich, 2003; Spinath \& Spinath, 2005).

Centrando-nos, agora, especificamente na variável atribuições causais, podemos referir que são crenças importantes que operam no fim da sequência motivacional e que influenciam as expetativas de sucesso futuro dos indivíduos. $\mathrm{Em}$ contextos educativos, à luz da perspetiva atribucional de Weiner (1985), os alunos procuram dar sentido às suas experiências acadêmicas, tentando explicar e compreender os seus bons e fracos resultados escolares. Os trabalhos de Weiner e colaboradores (Weiner, 1979; Weiner \& Kukla, 1970; Weiner \& cols., 1971; Weiner, Heckhausen, Myer \& Cook, 1972) 
revelam que os alunos utilizam as atribuições para explicar um resultado obtido e tais atribuições acabam por influenciar as suas emoções subsequentes e expetativas de desempenho futuro. Dito de outro modo, os resultados obtidos são influenciados pelas expetativas e reações afetivas que, por sua vez, influenciam a conduta de rendimento (intensidade, escolha de certas tarefas, esforço e persistência). No limite, podemos afirmar que não são os efeitos do êxito e fracasso em si mesmos que influenciam a conduta posterior, mas a valoração que cada sujeito lhes atribui (Weiner, 1985).

Na base da revisão bibliográfica apresentada, este artigo procura examinar o contributo específico do nível educativo familiar e das atribuições causais para 0 bom e fraco rendimento escolar no desempenho escolar dos alunos. Para o efeito, considera-se 0 rendimento nas disciplinas de matemática e de língua portuguesa. Esta análise, ao considerar alunos no final do ensino básico e do ensino secundánio, pretende verificar, ainda, se a importância relativa de ambas as variáveis se mantém constante ao longo desses dois níveis escolares.

\section{Participantes}

\section{Método}

A amostra deste estudo é formada por 2.082 alunos do $7^{\circ}$ ao $12^{\underline{0}}$ ano de escolaridade do ensino público de 11 escolas do continente e das regiões autônomas dos Açores e da Madeira. Os alunos repartem-se de uma forma relativamente equitativa por cada ano de escolaridade entre $07^{\circ}$ e $012^{\circ}$ ano de escolaridade. Desses alunos, 45,1\% (940) são rapazes e $54,9 \%$ (1.142) são raparigas. As idades oscilaram entre os 11 (11 alunos) e os 21 anos ( 2 alunos), situando-se a média de idades em 15,3 ( $\mathrm{DP}=1,84)$. Relativamente ao nível educativo familiar, 17\% (354) apresentam o $4^{\underline{0}}$ ano de escolaridade; $24,9 \%$ (519) 0 6 0 ano de escolaridade; $22 \%$ (458) o 90 ano de escolaridade; 21,1 (440) o $12^{\circ}$ ano de escolaridade; e 11,4 (238) o ensino superior. Importa acrescentar que 3,5\% (73) dos sujeitos não informaram o nível educativo dos pais.

\section{Instrumeto}

Para este estudo, foi utilizado o Questionánio de Atribuiçôes de Resultados Escdares na sua última versão (QARE; Miranda \& Almeida, 2008), que avalia as atribuições causais para as situações de bom e fraco desempenho acadêmico. Para cada uma dessas situações, apresentam-se 18 justificativas ou causas possíveis, devendo o aluno classificar cada uma delas de acordo com uma escala de tipo Liket com 5 pontos (desde 1, ou nunca, até 5, ou sempre). Do ponto de vista teórico, os itens do QARE repartem-se por três dimensões ou atribuições causais: duas reportadas a fatores pessoais, ou seja, atribuições à capacidade e atribuições ao empenho/método de estudo, e uma terceira dimensão reportada a contingências externas e aleatórias, por exemplo, à falta de incentivo/ incentivo por parte dos professores, nível de facilidade/ dificuldade dos testes, boa/ má sorte, ou ter/ não ter apoio afetivo dos pais. Essas três dimensões apresentam, ao longo dos vários estudos já realizados, índices de consistência interna nunca inferiores a 0,75 (Miranda \& Almeida, 2008).

\section{Procedimentos}

Para a presente investigação foi solicitada autorização prévia ao GabintedeEstatística ePlan€mento da Educação (GEPE) do Ministério da Educação (inquérito n. .0084100001 , registado em 30-12-2009, e aprovado em 7 de janeiro de 2010). Este questionário foi aplicado às turmas aleatoriamente selecionadas em cada escola durante um tempo letivo cedido pelos professores. Salientou-se aos alunos que os resultados se destinavam apenas a uma investigação, justificando a necessidade da sinceridade nas suas respostas. Não havendo boas e más respostas, pedia-se a leitura sempre atenta das possíveis causas e a sua ordenação de acordo com a escala dada. Possibilitava-se que os alunos não realizassem 0 preenchimento do questionário se assim o decidissem. Nenhum aluno tomou essa atitude, mas cerca de 400 protocolos foram anulados porque continham valores omissos. As análises estatísticas foram realizadas com recurso ao programa estatístico IBM SPSS versão 19.0.

\section{Resultados}

Na Tabela 1, apresentam-se as análises estatísticas descritivas (média e desvio padrão entre parênteses) das variáveis em estudo. A par dos valores nas dimensões atribucionais para as situações de bom e fraco desempenho escolar, descrevem-se também as classificações obtidas em língua portuguesa e em matemática, assim como o somatório das classificações dos alunos nessas duas disciplinas, e, ainda, a distribuição do nível educativo familiar, considerandose a habilitação escolar mais elevada de um dos progenitores. Importa, por último, referir que os resultados são apresentados separando os alunos por nível escolar (básico e secundário) em virtude da natureza curricular diferenciada. 
Tabela 1. Resultados nas atribuiçöes causais, nivel educativo familiar e classiticaçöes a portuguës e matemática

\begin{tabular}{|c|c|c|c|c|c|c|}
\hline \multirow[b]{2}{*}{ Variáveis } & \multicolumn{3}{|c|}{ Básico } & \multicolumn{3}{|c|}{ Secundário } \\
\hline & $\begin{array}{c}\text { Masculino } \\
\mathrm{N}=461\end{array}$ & $\begin{array}{c}\text { Feminino } \\
\mathrm{N}=420\end{array}$ & $\begin{array}{c}\text { Total } \\
\text { N=881 }\end{array}$ & $\begin{array}{l}\text { Masculino } \\
\mathrm{N}=479\end{array}$ & $\begin{array}{c}\text { Feminino } \\
\mathrm{N}=722\end{array}$ & $\begin{array}{c}\text { Total } \\
\mathrm{N}=1201\end{array}$ \\
\hline Nível educativo familiar & $8,6(3,14)$ & $8,4(3,20)$ & $8,5(3,17)$ & $8,6(3,26)$ & $8,3(3,24)$ & $8,4(3,26)$ \\
\hline Empenho e organização do estudo_BD & $3,2(0,71)$ & $3,6(0,66)$ & $3,4(0,71)$ & $2,9(0,72)$ & $3,4(0,63)$ & $3,2(0,71)$ \\
\hline is externas e aleatórias_ BD & $3,4(0,58)$ & $3,5(0,59)$ & $3,4(C$ & $3,0(0,56)$ & $3,0(0,54)$ & $3,0(0,55)$ \\
\hline Capacidades BD & $3,5(0,66)$ & $3,5(0,59)$ & $3,5(0,63)$ & $3,5(0,55)$ & $3,3(0,54)$ & $3,4(0,60)$ \\
\hline Empenho e organ & $2,7(0,81)$ & $2,5(0,87)$ & $2,6(0,85)$ & $2,9(0,84)$ & $2,6(0,78)$ & $2,7(0,82)$ \\
\hline Contingências externas e aleatórias $\mathrm{FD}$ & $2,5(0,80)$ & $2,3(0,87)$ & $2,4(0,84)$ & $2,4(0,72)$ & $2,2(0,68)$ & $2,3(0,71)$ \\
\hline Capacidades FD & $2,5(0,82)$ & $2,5(0,84)$ & $2,5(0,83)$ & $2,3(0,72)$ & $2,5(0,69)$ & $2,4(0,21)$ \\
\hline Português & $2,9(0,74)$ & $3,1(0,78)$ & $3,0(0,76)$ & $11,9(2,61)$ & $12,8(2,50)$ & $12,4(2,59)$ \\
\hline Matemática & $2,9(0,87)$ & $3,0(0,89)$ & $3,0(0,88)$ & $12,2(3,31)$ & $12,0(2,28)$ & $12,1(3,29)$ \\
\hline Português + Matemática & $5,8(1,44)$ & $6,2(1,53)$ & $6,0(1,49)$ & $24,2(5,34)$ & $25,0(5,23)$ & $24,6(5,3)$ \\
\hline
\end{tabular}

Legenda: BD = Bom Desempenho; FD = Fraco Desempenho

Em termos gerais, de acordo com a Tabela 1, pode-se referir que tanto os alunos do ensino básico como do ensino secundário tendem a apresentar como justificativas para o seu bom desempenho escolar as capacidades, e, para o fraco desempenho escolar, a falta de empenho e organização do estudo. Esses resultados sugerem uma tendência para explicar tanto, o bom desempenho como 0 seu fraco desempenho, recorrendo a causas internas, neste caso, à capacidade e ao esforço e organização do estudo.

Os resultados apontam que os alunos se responsabilizam tanto pelo bom como fraco desempenho escolar. Tomando o nível escolar familiar, a média das habilitações escolares familiares situa-se nos 8,5 anos de escolaridade sugerindo que a generalidade dos pais se situa num nível intermédio de escolaridade.

Na Tabela 2, apresentam-se as correlações entre as atribuições causais e nível educativo familiar com 0 rendimento dos alunos nas disciplinas de língua portuguesa matemática e a média nessas duas disciplinas, separadamente, por nível de ensino.

Tabela 2. Correlações entre as atribuições e nível educativo familiar com as classificações escolares dos alunos

\begin{tabular}{|c|c|c|c|c|c|c|}
\hline \multirow[b]{2}{*}{ Variáveis } & \multicolumn{3}{|c|}{ Ensino básico } & \multicolumn{3}{|c|}{ Ensino secundário } \\
\hline & $\begin{array}{c}\text { Língua } \\
\text { Portuguesa }\end{array}$ & Matemática & $\begin{array}{c}\text { L. Portuguesa } \\
+ \\
\text { Matemática }\end{array}$ & $\begin{array}{c}\text { Língua } \\
\text { Portuguesa }\end{array}$ & Matemática & $\begin{array}{c}\text { L. Portuguesa } \\
+ \\
\text { Matemática }\end{array}$ \\
\hline Nivel educativo familiar & $0,17 * *$ & $0,21 * *$ & $0,21 * *$ & $0,20^{* *}$ & $0,16^{* *}$ & $0,21 * *$ \\
\hline $\begin{array}{l}\text { Empenho_organizaçấo } \\
\text { do estudo_BD }\end{array}$ & $0,36^{* *}$ & $0,28^{* *}$ & $0,35^{* *}$ & $0,29 * *$ & $0,17^{* *}$ & $0,25^{* *}$ \\
\hline $\begin{array}{l}\text { Cont externas } \\
\text { aleatórias_BD }\end{array}$ & $0,16^{* *}$ & $0,14^{* *}$ & $0,16^{* *}$ & 0,04 & 0,06 & 0,06 \\
\hline Capacidad̄es_BD & $0,50 * *$ & $0,47 * *$ & $0,53 * *$ & $0,36 * *$ & $0,38 * *$ & $0,41 * *$ \\
\hline $\begin{array}{l}\text { Empenho_organização } \\
\text { do estudo_FD }\end{array}$ & $-0,23^{* *}$ & $-0,17^{* * *}$ & $-0,22^{* *}$ & $-0,15^{* *}$ & $-0,12^{* *}$ & $-0,15^{* *}$ \\
\hline $\begin{array}{l}\text { Cont externas e } \\
\text { aleatórias_FD }\end{array}$ & $-0,20 * *$ & $-0,19 * *$ & $-0,21 * *$ & $-0,17 * *$ & $-0,19 * *$ & $-0,21 * *$ \\
\hline Capacidad̄es_FD & $-0,29 * *$ & $-0,27 * *$ & $-0,31 * *$ & $-0,18 * *$ & $-0,23^{* *}$ & $-0,23 * *$ \\
\hline
\end{tabular}

Legenda: $\mathrm{BD}=$ Bom Desempenho; FD = Fraco Desempenho; ${ }^{* *} \mathrm{p}<0,01 ;{ }^{*} \mathrm{p}<0,05$

De modo geral, os coeficientes de correlação são baixos ou moderados, mesmo assumindo significado estatístico em virtude do tamanho da amostra. Por outro lado, verifica-se que os coeficientes de correlação assumem valores ligeiramente superiores junto dos alunos do ensino básico. As classificações em matemática e língua portuguesa correlacionam-se moderadamente com a atribuição causal à capacidade, sendo mais expressivas nos alunos do ensino básico. As atribuições à falta de capacidade para 0 fraco desempenho escolar associam-se ao rendimento em língua portuguesa tanto no ensino básico como no ensino secundário, sendo tais associações ligeiramente mais fortes no ensino básico. As correlações mais expressivas verificam-se entre a atribuição à capacidade para o bom desempenho e 0 fraco desempenho escolar. Pode-se ainda referir que o nível educativo dos pais está associado positivamente ao rendimento escolar dos alunos, como seria expectável em face da literatura na área. 
Para apreciar o contributo específico do nível educativo familiar e das atribuições causais dos alunos na explicação do seu rendimento escolar, procedeu-se uma análise de regressão com procedimento stepvise Por razões de parcimônia, considerou-se a média nas classificações dos alunos nas disciplinas de língua portuguesa e matemática. $O$ modelo mostra-se estatisticamente significativo para 0 ensino básico $(\mathrm{F}=87,003 ; \quad \mathrm{p}<0,00)$ e para 0 ensino secundário $(F=54,223 ; p<0,001)$.

Na Tabela 3, sintetizou-se a informação recolhida nesta análise da regressão. As atribuições causais: capacidade para o bom desempenho, capacidade para 0 fraco desempenho, empenho e organização do estudo para 0 bom desempenho escolar, contingências externas e aleatórias para o bom desempenho escolar, bem como o nível educativo familiar, explicam, no seu conjunto, 34,5\% da variância do rendimento escolar dos alunos do ensino básico. Os valores obtidos sugerem, ainda, que a variável nível educativo familiar acrescenta apenas $1 \%$ da variância do rendimento escolar, sendo a maior parte desta explicada pela atribuição à capacidade para 0 bom desempenho escolar $(28 \%)$.

Tabela 3. Resultados da análise da regressão do rendimento escolar por nível escolar

\begin{tabular}{|c|c|c|c|c|c|}
\hline Nível escolar & Modelo & $\mathrm{R}^{2}$ Ajust & Beta & $\mathrm{t}$ & Prod \\
\hline \multirow[t]{5}{*}{ Básico } & Cap BD & 0,280 & 0,414 & 11,343 & 0,000 \\
\hline & Cap_BD + Cap_FD & 0,305 & $-0,164$ & $-5,462$ & 0,000 \\
\hline & Cap BD + Cap FD + Emp Org Estu BD & 0,317 & 0,230 & 5,983 & 0,000 \\
\hline & Cap BD + Cap FD + Emp Org Estu BD +Cont Ext Aleat BD & 0,335 & $-0,168$ & $-4,627$ & 0,000 \\
\hline & $\begin{array}{l}\text { Cap_BD + Cap_FD + Emp_Org_Estu_BD + Cont_Ext_Aleat_BD } \\
+ \text { N_Ed Familiar }\end{array}$ & 0,345 & 0,101 & 3,451 & 0,001 \\
\hline \multirow[t]{6}{*}{ Secundário } & Cap_BD & 0,134 & 0,285 & 9,018 & 0,000 \\
\hline & Cap_BD + Emp_Org_Estu_BD & 0,165 & 0,204 & 6,577 & 0,000 \\
\hline & Cap_BD + Emp_Org_Estu_BD + N_Ed_Familiar & 0,188 & 0,139 & 4,922 & 0,000 \\
\hline & Cap_BD + Emp_Org_Estu_BD + N_Ed_Familiar + Cap_FD & 0,202 & $-0,074$ & $-2,165$ & 0,031 \\
\hline & $\begin{array}{l}\text { Cap_BD + Emp_Org_Estu_BD + N_Ed_Familiar + Cap_FD + } \\
\text { Cont Ext Aleatórias BD }\end{array}$ & 0,208 & $-0,099$ & $-3,316$ & 0,001 \\
\hline & $\begin{array}{l}\text { Cap_BD + Emp_Org_Estu_BD + N_Ed_Familiar + Cap_FD + } \\
\text { Cont Ext Aleatórias BD + Cont Ext Aleatórias FD }\end{array}$ & 0,213 & $-0,092$ & $-2,828$ & 0,005 \\
\hline
\end{tabular}

Legenda: BD = Bom Desempenho; FD = Fraco Desempenho

No ensino secundário, as atribuições causais (capacidade para o bom desempenho, empenho e organização do estudo para 0 bom desempenho escolar, capacidade para 0 fraco desempenho, contingências externas e aleatórias para 0 bom desempenho escolar e contingências externas e aleatórias para o fraco desempenho escolar) explicam $19 \%$ da variância do rendimento escolar, explicando 0 nível educativo familiar 2,3\% da variância. Também nesse nível de ensino, a variável capacidade para o bom desempenho escolar explica a maior percentagem da variância do rendimento $(13,4 \%)$.

\section{Discussão}

Tanto os alunos do ensino básico como do ensino secundário apresentam como justificativa para o seu bom desempenho escolar as capacidades e para o seu fraco desempenho a falta de empenho e organização do estudo. Este padrão atribucional, assente em causas internas, adaptativo e promotor do sucesso escolar é também observado noutros estudos (Almeida, Miranda \& Guisande, 2008; Boruchovitch, 2004; Ferreira \& cols., 2002; Miranda, Almeida \& Almeida, 2007). Constata-se ainda que, com 0 avançar na escolaridade, os alunos assumem maior responsabilidade pelos seus desempenhos escolares, sejam positivos ou negativos, desaparecendo alusões aos fatores sorte ou professor (Boruchovitch, 2004; G onzaga, Morais, Santos \& Jesus, 2006).

Por sua vez, as correlações obtidas sugerem uma associação positiva entre atribuições causais internas e nível educativo dos pais com o rendimento acadêmico, sobretudo junto dos alunos do ensino básico. Esses coeficientes reforçam que variáveis motivacionais e familiares são relevantes para um maior entendimento da aprendizagem e do rendimento escolar e devem ser examinadas em conjunto, uma vez que estes mesmos índices emergem na análise de regressão (Boekaerts, 1997; Broman \& cols., 1985; Jadue, 2003; Jadue \& cols., 2005; Nunes, 1990).

O potencial poder explanatório das atribuições causais na compreensão dos fatores associados ao rendimento escolar, sobretudo quando se trata do fator capacidade, foi, sem dúvida, um dado interessante encontrado no presente estudo. Entretanto, recomenda-se que 0 impacto das atribuições de causalidade no desempenho escolar e na aprendizagem possa ser melhor aquilatado por novas pesquisas, futuramente, já que este trabalho possibilitou apenas 
uma identiticaçäo das causas empregadas pelos alunos em suas interpretações de sucesso e fracasso escolar, não se tendo acesso, de fato, aos aspectos que concorrem diretamente para a aprendizagem.

Se, por um lado, as variáveis estudadas no presente estudo explicam inegavelmente uma importante parte da variância do rendimento acadêmico, sobretudo junto dos alunos do ensino básico, por outro, lançam questões importantes para a agenda da pesquisa futura no que concerne à necessidade de maior compreensão acerca ao papel do nível educativo, por exemplo, da mãe: seria ele mais determinante como explicação do desempenho do que o grau de escolarização ou a habilitação acadêmica do pai? Outro ponto importante a ser investigado em estudos futuros é se, de fato, a escolaridade dos pais é mais relevante como fator para 0 entendimento dos aspectos relacionados ao desempenho escolar bemsucedido do que o nível socioeconômico, tal como defendem alguns estudiosos do tema (Davis-Kean, 2005; Formosinho, 1991; González, 2001; Mella \& Ortiz, 1999). Diferenças de gênero nas atribuições causais merecem também ser exploradas em novos estudos, já que é possível que rapazes e raparigas forneçam explicações distintas para suas experiências de sucessos e fracasso no contexto educativo. Uma outra variável que urge ser examinada pela pesquisa futura é as atribuições de professores acerca do desempenho de seus alunos e seu real impacto no desempenho deles (Bzuneck \& Sales, 2011).

\section{Considerações finais}

A investigação nas últimas décadas destaca 0 impacto das variáveis motivacionais na explicação da aprendizagem e do rendimento escolar dos alunos. Nesse sentido, as teorias cognitivo-motivacionais ou sociocognitivistas propõem que a motivação se constitui como um processo de mediação cognitiva entre 0 sujeito e 0 seu desempenho na tarefa, traduzindo-se em sentimentos de autoeficácia atribuições causais, expetativas ou metas. Nesta linha, reforça-se a sua relevância para a aprendizagem e desempenho escolar, parecendo que a sua importância aumenta à medida que se avança na escolaridade (Downing \& cols., 1977; Elliot, 2006; García \& Sánchez, 2005; G onzalez-Pienda \& cols., 2002).

Os resultados deste estudo apontam a importância de causas atribucionais internas tanto para a justificativa do bom como do fraco desempenho acadêmico, nomeadamente a referência pelos alunos à capacidade e ao esforço. Estas atribuições apresentamse bastante pertinentes nas situações de sucesso escolar. Por norma, tais atribuições associam-se a sentimentos de eficácia quando o aluno alcança o ëxito. Situação inversa ocorre quando 0 aluno associa 0 seu fraco rendimento a uma baixa capacidade, necessitando nessa altura da ajuda dos professores ou encarregados de educação no sentido de reinterpretar sua experiência, reatribuindo à falta de esforço e/ ou método de estudo inadequado a razão das suas dificuldades. Esforço e método de estudo são atribuições causais suscetíveis de serem entendidas como modificáveis e, também, controláveis pelo próprio aluno.

Ao mesmo tempo, a investigação aponta que a realidade sociocultural da família está associada com 0 rendimento escolar dos alunos. Pais com melhores níveis sociais e acadêmicos envolvem-se mais na educação e nas tarefas escolares dos seus filhos, estimulando-os na adoção de métodos de estudo mais apropriados. Por outro lado, a pesquisa na área sugere que os pais são poderosos socializadores da autoperceção que as crianças têm do seu desempenho escolar e dos níveis de motivação que colocam nas suas aprendizagens (Jacobs \& Eccles, 1992; Maluf \& Bardelli, 1984; McGrath \& Repetti, 2000; Neves \& Almeida, 1996). No presente estudo, os níveis acadêmicos dos pais correlacionam-se com 0 rendimento escolar dos alunos; contudo, na análise de regressão, o seu peso passa despercebido pela maior relevância das atribuições causais.

Considerando a importância constatada no presente estudo no que concerne à associação dos fatores motivacionais e familiares na compreensão do desempenho escolar de estudantes, bem como o fato de 0 presente estudo ter se restringido à investigação das atribuições causais e do nível educativo familiar, espera-se que maiores esforços sejam direcionados a pesquisas com delineamentos multivariados, que examinem a força conjunta de outras variáveis. A identificação de um conjunto alargado de variáveis e a compreensão de como se combinam na explicação da aprendizagem e do rendimento acadêmico continuam a ser objetivos centrais da psicologia escolar por forma a contribuir para uma aprendizagem de qualidade e para um desempenho escolar bem-sucedido.

\section{Referências}

Almeida, L. (1996). Cognição e aprendizagem: como a sua aproximação conceptual pode favorecer 0 desempenho cognitivo e a realização escolar. Psicdọa: Teria, Investicgacão ePrática, 1, 17-32.

Almeida, L., \& Roazzi, A. (1988). Insucesso escolar: insucesso do aluno ou insucesso do sistema escolar. Reuista Partuguesa deEducaçãa, 1, 14-35. 
Almeida, L. S., Miranda, L., \& Gisande, A. (2008). Atribuições causais para o sucesso e fracasso escolares em função do gênero e do ano escolar. EstudosdePsicdoga, 25, 169-184.

Ames, C. (1993). Parent invdvement: The reationship between shod-tohme commmication and parents perceptions and beies (Rep. no. 15). Lansing: Michigan State University, Center on Families, Communities, Schools and Children's Learning.

Barbera, C. G. (2003). Fatores deteminantes de bajo rendimiento acadérico en eduraaón seundania (Tese de D outorado). Universidad Complutense de Madrid, Espanha.

Barros, A. M. (1996). Atribuiçês causais e expetativas de contrdo do desempenho na matemática (Tese de D outorado). Universidade do Minho, Braga, Portugal.

Boekaerts, M. (1997). Self-regulated learning: a new concept embraced by researchers, policy makers, educators, teachers, and students. Leaming and Instruction, 7, 161-186.

Boruchovitch, E. (1994). As variáveis psicológicas e 0 processo de aprendizagem: uma contribuição para a Psicologia Escolar. Psicdogia: Temia ePesquisa, 10, 129-139.

Boruchovitch, E. (2004). A auto-regulação da aprendizagem e a escolarização inicial. Em E. Boruchovitch \& J. A. Bzuneck (Orgs), Aprendizagm procesos psicdóg்os e o contexto soial ma eda (pp. 37-60). Petrópolis, Rio de Janeiro: Vozes.

Broman, S., Bien, E., \& Shaugenessy, P. (1985). Low adieing dildren: The first seven years Hillsdale, NJ: Lawrence Erlbaum.

Bzuneck, J. A., \& Sales, K. F. S. (2011). Atribuições interpessoais pelo professor e sua relação com emoções e motivação do aluno. PsiøUSF, 16(3), 307-315.

Davis-Kean, P. E. (2005). The influence of parent education and family income on child achievement: the indirect role of parental expectations and the home environment. Jaumal of Family Psydhdogy, 19(2), 294-304.

Degenszajn, R. D., Roz, D. P., \& Kotsubo, L. (2001). Fracasso escolar: uma patologia dos nossos tempos? Peeliatria (SãoPaulo), 1, 106-113.

Deslandes, R. (2001). A vision of home-school partnership: three complementary conceptual frameworks. Em F. Smith, K. Van der Wolf \& P.
Sleegers (Eds.), A bridge to the future Cdlaboration between parents, schods and commuities (pp. 11-23). Nijmegen: ITS.

Downing, J., Ollila, L. L., \& Oliver, P. (1977). Concepts of language from differing socioeconomic backgrounds. The Jaumal of Edurational Resch, 70(5), 277-281.

Dumka, L. E., Gonzales, N. A., Wheeler, L. A., \& Millsap, R. E. (2010). Parenting self-efficacy and parenting practices over time in Mexican American families. Jaumal of Family Psychilog, 24(5), 522-531.

Elliot, A. J. (2006). The hierarchical model of approach avoidance motivation. Mdivation and Emotion, 30, 111-116.

Engin-Demir, C. (2009). Factors influencing the academic achievement of the Turkish urban poor. Intemational Jaumal of Edurational Deddqumet, 29, 17-29.

Eurydice (1994). Measures to combat shod failure A dallenge for the construction of Europe Bruxelas: Comissão Europeia.

Ferreira, M. C., Assmar, E. M. L., O mar, A. G., Delgado, H. H., González, A. T., Souza, M. A., \& Cisne, M. C. F. (2002). Atribuição de causalidade ao sucesso e fracasso escolar: um estudo transcultural Brasil-Argentina-México. Psicdogia: RellexãoeCńtica, 15, 515-527.

Flouri, E., \& Buchanan, A. (2004). Early father's and mother's involvement and child's later educational outcomes. British Jaumal of Educational Psychdogy, 74, 141-153.

Formosinho, J. (1991). A igualdade em educação. Em J. Formosinho, A. S. Fernandes \& E. L. Pires (O rgs.), A construcãa soial da elucação ecdar (pp. 169-186). Coleção Biblioteca Básica de Educação e Ensino. Rio Tinto: Edições ASA.

Garcia, J. A., \& Sánchez, J. M. R. (2005). Prácticas educativas familiares $\mathrm{y}$ autoestima. Psicethema, 17(1), 76-82.

Garfaella, P. R., Gargallo, L. B., \& Sánchez, F. (2001). Medidas y estrategias para la reducción del absentismo escolar. Revista de Estudios de Junentud, 52, 27-36.

Gonzaga, L., Morais, S., Santos, J., \& Jesus, S. N. (2006). Atribuições causais do sucesso e do fracasso académico: estudo comparativo de estudantes do ensino secundánio e do superior. Em C. Machado, L. Almeida, M. A. Guisande, M. 
8 Miranda, L. C. \& Cds. Atribuições causais, nível educativo familiar e desempenho escolar em alunos portugueses

Gonçalves \& V. Ramalho (Urgs.), Adas da XI Confê̂nia Intemadional de Avaliação Psiclógica: Fomas e Contextos (pp. 951-960). Braga: Psiquilíbrios.

González, D. S. (2001). Influenia de los fatores soieconómicos en e rendimientoesclar intemadional: hada la igualdad de qpartunidades ecturativas Recuperado: Janeiro de 2011. Disponível: http/ / unuruamed BUCM/ @ do/ 0101/ 0101.PDF.

González-Pienda, J. A., Núñez, J. C., GonzálezPumariega, S., Álvarez, L., Roces, C., \& García, M. (2002). A structural equation model of parental involvement, motivational and aptitudinal characteristics, and academic achievement. The Jaumal of Expeimental Education, 70(3), 257-287.

Guzzo, R. S. L. (2001). Saúde psicológica, sucesso escolar e eficácia da escola: desafios do novo milênio para a psicologia escolar. Em Z. A. P. Del Prette (O rg.), Psicoloja esclar e elucacional, saúde e qualidadedevida: explorandofronteiras (vol. 1, pp. 2542). Campinas, SP: Alínea.

Jacobs, J., \& Eccles, J. (1992). The impact of mothers' gender-role stereotypic beliefs on mothers' and children's ability perceptions. Jaumal of Pessonality and Social Psychdoy, 63, 932-944.

Jadue, G. (2002). Fatores psicológicos que predisponen al bajo rendimiento, al fracaso y a la deserción escolar afectan el rendimiento escolar. Estudios Peelagóǵos, 28, 193-204.

Jadue, G. (2003). Transformaciones familiares en Chile: riesgo creciente para el desarrollo emocional, psicosocial y la educación de los hijos. Estudios Peelagóg்os 29, 115-126.

Jadue, G., Galindo, A., \& Navarro, N., L. (2005). Fatores protectores y fatores de riesgo para el desarrollo de la resiliência encontrados en una comunidad educativa en riesgo social. Estudios Peclagóg்os 31(2), 43-55.

Maluf, M. R., \& Bardelli, C. (1984). Que valor o professor atribui à capacidade e resultado obtido pelos alunos em contexto de realização escolar? Arquivos Brasileiros dePsidogia, 36(4), 132-411.

Martin, J., Ranson, S., \& Tall, G. (1997). Parents as partners in assuring the quality of schools. Scettish Education Reiew 29(1), 39-55.

McGrath, E., \& Repetti, R. (2000). Mothers' and fathers' attitudes toward their children's academic performance and children's perceptions of their academic competence. Jaumal of Yath and Addescence, 29(6), 713-723.

Mella, O., \& Ortiz, I. (1999). Rendimiento escolar. Influencias diferenciales de fatores externos e internos. Reista Latinommicana de Estudios Edurativos, 29(1), 69-92.

Mendonça, A. (2007). A problemática doinsuresso esclar - a esclaridade darigatónia mo arquipáago da Madkira em finais do séulo XX (Tese de Doutorado). Universidade de Madeira, Madeira, Funchal, Portugal.

Miranda, L. C., \& Almeida, L. S. (2008) Questionánio das Atribuiç̃es Causais para os Resultados Escdares (QARE). Braga: Universidade do Minho.

Miranda, L., Almeida, L. S., \& Almeida, A. (2007). Estudo das relações entre as atribuições para os resultados escolares o rendimento na matemática, língua portuguesa e o ano escolar. Em A. Barca, M. Peralbo, A. Porto, B. D. Silva \& L. Almeida (Eds.), Adas do IX Congreso Intemadional Galego Potuguês dePsicopedagoxia (pp. 2937-2946). Coruña: Universidade da Coruña.

Neves, M. B. J., \& Almeida, S. F. C. (1996). 0 fracasso escolar na 5a série, na perspectiva de alunos repetentes, seus pais e professores. Psicdoja: Teria epesquisa, 12, 147-156.

Nunes, A. N. A. (1990). Fracasso escolar e desamparo adquirido. Psicologa: Teria ePesquisa, 6, 139-154.

OECD (2009). Education at a gance OECD indicatars Paris: OECD.

Pérez-Díaz, V., Rodríguez, P., J., C., \& Sánchez, F., L. (2001). La família españda ante la educadón de sus hijos Barcelona: Fundación La Caixa.

Pintrich, P. R. (2003). A motivational science perspective on the role of student motivation in learning and teaching contexts. Joumal of Educational Psychdogy, 95, 667-686.

Reeve, J., Deci, E. L., \& Ryan, R. M. (2004). Selfdetermination theory: A dialectical framework for understanding socio-cultural influences on student motivation. Em D. M. McInerney \& S. Van Etten (Eds.), Big theeries revisited (pp. 31-60). G reenwich, CT: Information Age Press.

Spinath, B., \& Spinath, F. M. (2005). Longitudinal analysis of the link between learning motivation and competence beliefs among elementary school children. Leamingand Instruttion, 15, 87-102. 
Symeou, L. (2005). Past and present in the notion of school-family collaboration. AulaAbieta, 85, 165184.

UNESCO . (1996). Eduração umtesaro a descobir. Porto: Edições ASA.

Weiner, B. (1979). A theory of motivation for some classroom experiences. Jaumal of Educational Psychdogy, 71, 3-25.

Weiner, B. (1985). An attributional theory of achievement motivation and emotion. Psychdogcal Review 92, 548-573.

Weiner, B., \& Kukla, A. (1970). An attributional analysis of achievement motivation. Jaumal of Personalityand Social Psydhdogy, 5, 1-20.
Weiner, B., Frieze, I., Kukla, A., Reed, L., Rest, S., \& Rosenbaum, R. M. (1971). Perceiving the causes of success and failure. Em E. E. Jones. D. E. Kanhouse, H. H. Kelley, R. E. Nisbett, S. Valins \& B. Weiner (Eds.), Attribution: Peciving the causes of behavior (pp. 95-120). Morristown, NJ: General Learning Press.

Weiner, B., Heckhausen, H., Meyer, W., \& Cook, R. E. (1972). Causal ascriptions and achievement behavior: A conceptual analysis of locus of control. Jaumal of Pessanality and Social Psychdogy, 21, 239-248.
Reedbidoem10/ 06/ 2011
Refamuladoem05/ 03/ 2012
Aprovadbem06/ 03/ 2012

Sobre os autores:

Lúcia C. Miranda é psicóloga pela Universidade de Coimbra, PhD pela Universidade do Minho em Psicologia área de especialização Psicologia da Educação, Mestre em Ciências da Educação, na área de Psicologia da Educação pela Universidade de Coimbra. Professora Auxiliar no Instituto Superior de Educação e Trabalho- Porto. Investigador colaborador no Centro de Investigação em Educação (CIEd) na Universidade do Minho.

Leandro S. Almeida é psicólogo pela Universidade do Porto, $\mathrm{PhD}$ pela Universidade do Porto, em Psicologia (Psicologia da Educação), Agregação em Psicologia da Educação pela Universidade do Minho, onde exerce funções de Professor Catedrático. D ocência e investigação nas áreas da cognição e da aprendizagem. Coordena alguns projetos de investigação na área da sobredotação na população estudantil e da excelência na área profissional em adultos, centrados na avaliação e na intervenção.

Evely Boruchovitch é psicóloga pela Universidade do Estado do Rio de Janeiro. Ph.D em Educação pela University of Southern Califórnia. Pós-doutorado em Psicologia Educacional pela Faculdade de Educação da Unicamp e em Avaliação Psicológica pelo Programa de Pós-graduaçao em Psicologia da USF. Professora Titular do Departamento de Psicologia Educacional e do Programa de Pós-graduação em Educação da Faculdade de Educação da Unicamp. Membro do Grupo de Estudos e Pesquisas em Psicopedagogia (G epesp-Unicamp). Bolsista produtividade do CNPq.

António R. Almeida é licenciado em Ensino da Matemática pela Universidade Portucanse, Mestre em Matemática especialização em Ensino da Matemática pela Universidade Portucalense. Professor do Ensino Básico e Secundário na Escola Secundária de Celorico de Basto.

Sofia A. Abreu é licenciada em Desporto e Educação Física pela Universidade do Porto, Mestre em Ciências do Desporto com especialização em Desporto para Crianças e Jovens, pela Faculdade de Ciências do Desporto e de Educação Física, da Universidade do Porto. Professora do Ensino Básico e Secundário na Escola Básica de 2ํe 3ํㅡㄴ Ciclos de Bocage.

Contato com os autores:

Instituto Superior de Educação e Trabalho - Porto; R. Pereira Reis no 399, 4200-448. Porto.

Email: lrcmiranda@gmail.com 